\title{
La complejidad de articular derechos: alimentación y cuidado
}

\author{
The complexity of articulating rights: nutrition \\ and care
}

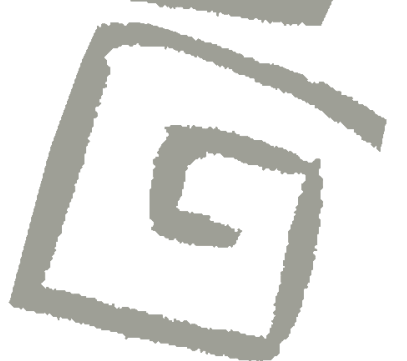

Laura Cecilia Pautassi ${ }^{1}$

${ }^{1}$ Doctora en Derecho Social. Investigadora independiente, Consejo Nacional de Investigaciones Científicas y Técnicas. Investigadora, Instituto de Investigaciones Jurídicas y Sociales Ambrosio L. Gioja, Facultad de Derecho, Universidad de Buenos Aires, Argentina.
RESUMEN El artículo analiza la tensión existente entre el reconocimiento de derechos de las personas, en especial el derecho a la alimentación adecuada tal como se expresa en pactos y tratados internacionales de Derechos Humanos, y la escasa vinculación con el cuidado, entendido como el conjunto de actividades indispensables para satisfacer las necesidades básicas de la existencia y reproducción humana y social. Aplicando la metodología del enfoque de derechos y de género, el artículo aborda, por una parte, el alcance del derecho a la alimentación y el impacto a nivel de institucionalidad pública $y$, por el otro, el reconocimiento reciente del cuidado como derecho a nivel regional y la invisibilización persistente en las políticas públicas. Los resultados obtenidos permiten presentar una agenda de investigación y acción que identifica tensiones y oportunidades para lograr la universalización en el ejercicio de derechos a partir de políticas públicas integrales e interdependientes.

PALABRAS CLAVES Alimentación; Derechos Humanos; Convenios Internacionales; Políticas Públicas.

\begin{abstract}
This article analyzes the existing tensions between the recognition of human rights - especially the right to adequate food as it is defined in international agreements and treaties - and the insufficient connection made with care, understood as the set of activities necessary to satisfy the basic needs of existence and human and social reproduction. Applying a methodological approach based in rights and gender, the article analyzes, on one hand, the scope of the right to food and its impact at the level of public institutionality, and on the other, the recent recognition of care as a right at a regional level and its persistent invisibilization in public policies. The results obtained allow for a research and action agenda that identifies tensions and opportunities to achieve universalization in the exercise of rights based in comprehensive and interdependent public policies.
\end{abstract}

KEY WORDS Feeding; Human Rights; International Agreements; Public Policies. 


\section{DERECHOS AL ALCANCE DE LA POLÍTICA PÚBLICA}

Pensar la alimentación, en tanto práctica individual, familiar o comunitaria conlleva una relación necesaria con el cuidado, en toda su dimensión, la cual se encuentra a su vez atravesada por diversas estrategias, experiencias y consumos que redundan en las condiciones de vida y resolución de las necesidades básicas, que van a impactar directamente en satisfactores diversos y dar cuenta de la "complejidad del evento alimentario"(1). Sin embargo, como todo lo relacionado con el cuidado se encuentra invisibilizado, asociado al trabajo no remunerado que realizan principalmente las mujeres en sus hogares, destinado a los integrantes del núcleo familiar, su relevancia no es reconocida y se ocultan las interrelaciones entre uno y otro, incluso en los casos en que el cuidado se encuentra mercantilizado ${ }^{(2)}$.

El acto de "cuidar" incluye una diversidad de acciones que van a "sostener" a una persona, la van a "nutrir", lo cual incluye un conjunto amplio de actividades indispensables para satisfacer las necesidades básicas de la existencia y reproducción humana, y ofrece elementos físicos, simbólicos y emocionales que posibilitan la vida en sociedad, todo lo cual sitúa al cuidado en una indiscutible centralidad. Estas acciones incluyen desde el autocuidado, el cuidado directo de otras personas -que se traduce como una actividad interpersonal e intensiva de cuidado-, la provisión de las condiciones previas y concomitantes con que se realiza el cuidado -la compra y preparación de alimentos, el pago de servicios, el lavado de la ropa- y la gestión del cuidado -coordinar horarios, realizar traslados a centros educativos y a otras instituciones, preparar viandas, supervisar el trabajo de la cuidadora remunerada-, entre tantas otras actividades que demanda. El cuidado, por lo tanto, permite atender las necesidades de las personas dependientes, por su edad o por sus condiciones/capacidades (niños, niñas y adolescentes, personas mayores, enfermas o con discapacidades) y también de las personas que podrían autoproveerse dicho cuidado ${ }^{(3,4)}$.

Estos actos, sean aislados o coordinados, se traducen en una particular forma de organización social del cuidado, que desde la economía feminista se ha definido como la forma en que interrelacionadamente las familias, el Estado, el mercado y las organizaciones comunitarias, producen y distribuyen cuidado. La noción de organización social del cuidado se encuentra relacionada con el diamante de cuidado $^{(5)}$ analizada también desde el estudio de los regímenes de bienestar como el reemplazo de la otrora "triada del bienestar": Estado-mercado-familias $^{(6)}$ como representación de la ingeniería a través de la cual se provee el cuidado y la responsabilidad de cada ámbito. Así, el diamante de cuidado -que Evers et al.(7) denominan diamante del bienestar- exhibe la presencia de cuatro actores, pero también las dependencias mutuas que se establecen entre ellos: la provisión de cuidados no ocurre de manera aislada o estanca, sino que resulta de una continuidad en la que se suceden trabajo, actividades, ocio, responsabilidades y obligaciones.

En relación con estas últimas, si bien los albores del siglo XXI se han caracterizado por el arribo del "enfoque de derechos", como metodología que los sistemas protectorios internacionales y regionales de derechos humanos ofrecen a los Estados como herramienta para implementar en su accionar, los resultados distan de ser los esperados. Es decir, el denominado "enfoque", en tanto metodología transversal establece formas precisas de interpretación del alcance de las obligaciones contenidas en los pactos y tratados internacionales de Derechos Humanos y, a su vez, incluye aquellos estándares interpretativos desarrollados por los órganos de monitoreo y vigilancia internacional (como los comités de los pactos y tratados, la labor de los relatores para cada derecho humano y, en el caso del sistema regional de derechos humanos, las sentencias de la Corte Interamericana de Derechos Humanos, los informes y medidas adoptadas por la Comisión Interamericana de Derechos Humanos, y el monitoreo de los grupos de seguimiento de 
las convenciones de "las Américas" de la Organización de Estados Americanos).

Normas internacionales, interpretación, sentencias, medida adoptadas, observaciones generales sobre el cumplimiento de derechos conforman un corpus interpretativo, cuyo objetivo consiste en precisar e identificar el alcance de los derechos comprometidos, dado que deben ser incorporados en el diseño, implementación y evaluación de las políticas públicas. Por lo tanto, el enfoque le recuerda a los Estados Parte que las obligaciones ratificadas no resultan un anhelo político o una voluntad de paz y armonía regional, sino precisamente enfatiza que el mandato estatal consiste en "atravesar" sus políticas, acciones de gobierno, la legislación, y que las sentencias de juezas y jueces deben basarse en el reconocimiento y garantía de los derechos de cada persona. Este atravesamiento implica implementar sistemas protectorios, garantías efectivas y satisfacción plena de derechos civiles y políticos, y de derechos económicos, sociales y culturales (DESC) reconocidos a nivel constitucional e internacional. No es suficiente que algunos derechos estén enunciados o incorporados, sino que requiere la universalidad, integralidad e interdependencia en la satisfacción de todos y cada uno de estos derechos.

$Y$ allí es donde se encuentra el nudo crítico, especialmente, en el caso de América Latina, el lenguaje de derechos ha invadido la esfera gubernamental: los decisores políticos anuncian planes y políticas sociales con un marco de derechos, las organizaciones sociales incluyen un reconocimiento activo y "lucha por derechos", la ciudadanía ha recuperado mayor fuerza y un creciente empoderamiento en torno al ejercicio de derechos, los tribunales locales han ido reconociendo este marco y una nueva jurisprudencia impacta en las políticas sociales locales. Sin embargo, el balance señala que el enfoque de derechos todavía no ha "atravesado" la institucionalidad pública y se mantiene aún a nivel retórico.

En el caso de la alimentación, cabe destacar que ha sido reconocida como derecho humano desde los inicios de los sistemas protectorios, tanto en la Declaración Universal de Derechos Humanos, que dispone que toda persona tiene derecho a un nivel de vida adecuado que le asegure la salud y el bienestar, con particular mención a la alimentación, en el Pacto Internacional de Derechos Económicos, Sociales y Culturales de 1966, en el que se reconoce el derecho de todos a una alimentación adecuada, al señalar en el Artículo 11 como obligaciones estatales el deber de garantizar el "derecho de toda persona a un nivel de vida adecuado para sí y su familia, incluso alimentación, vestido y vivienda adecuados y a una mejora continua de las condiciones de existencia"(8), y al incorporar explícitamente el derecho fundamental de toda persona a no pasar hambre. como en la Declaración Universal sobre la Erradicación del Hambre y la Malnutrición de 1974. En el caso del sistema interamericano de Derechos Humanos (SIDH), la Convención Americana de Derechos Humanos y, específicamente, el Protocolo Adicional de la Convención Americana en materia de Derechos Económicos, Sociales y Culturales "Protocolo de San Salvador", en sus Artículos 12 y 17 , reconoce explícitamente el derecho humano a la alimentación adecuada.

En el año 2000, en el marco de la Declaración del Milenio, los 189 países firmantes se comprometieron a la erradicación de la pobreza extrema y el hambre, entre otros Objetivos de Desarrollo del Milenio que fueron evaluados en la ronda 2015 y fortalecidos en la agenda 2030, a partir de adoptar 17 Objetivos de Desarrollo Sostenible y 169 metas para su cumplimiento. Así, el Objetivo 2 se propone "poner fin al hambre, lograr la seguridad alimentaria y la mejora de la nutrición y promover la agricultura sostenible" (9) junto con el compromiso de eliminar la pobreza en todas sus formas, sumando consensos para avanzar en la construcción de una agenda regional basada en la igualdad. En cambio, el activismo en torno al reconocimiento del cuidado ha sido débil, a pesar de las tensiones que su resolución acarrea para las familias y la sobrecarga de trabajo que implica para las mujeres. Mucho menor es la denuncia de la injusta división sexual del trabajo, que afecta centralmente a 
las mujeres, en todos los ámbitos de la vida en los que se desempeñen. Si bien se ha realizado un avance importante en esta agenda en torno al Objetivo 5 "Lograr la igualdad de género y empoderar a todas las mujeres y las niñas"(9), y se contemplan metas específicas para poner fin a todas las formas de discriminación y de violencia contra las mujeres y las niñas, asegurar el acceso universal a la salud sexual y los derechos reproductivos, abordar las necesidades de nutrición de las adolescentes y las embarazadas (meta 2.2) y reconocer y valorar los cuidados y el trabajo doméstico no remunerado (meta 5.4), sigue sin integrar los consensos centrales en torno al desarrollo.

Curiosamente, el cuidado hasta hace muy pocos años no había sido reconocido como derecho humano, sino que solo se incluía en relación con prestaciones vinculadas a personas enfermas o adultas mayores $\mathrm{O}$, en otros casos, se reconocía el derecho a la seguridad social vinculado a la maternidad, exclusivamente en el marco de la inserción asalariada formal de las mujeres trabajadoras, como otras medidas de conciliación trabajofamilia, pero solo en las mujeres, restringiendo un ejercicio activo a los varones. El punto que quiero recuperar es que en el reconocimiento del derecho a la alimentación, su traducción normativa internacional es indiscutible como lo es también a nivel constitucional, sumado a la prolífica producción legislativa de los últimos años en América Latina, con leyes marco y otras medidas de corte integral e incluso, en muchos países, con la recuperación de valores culturales y étnicos en torno a la cultura alimentaria. Sin embargo, es aún escaso el anclaje en políticas alimentarias universales y respetuosas del conjunto de derechos humanos. Por el contrario, la profusión de programas y medidas focalizadas para garantizar programas nutricionales es notoria, dejando de lado los estándares de universalidad e interdependencia de los derechos humanos.

En el otro extremo, el reconocimiento del cuidado como un derecho humano, no ha sido planteado sino hasta muy recientemente, con la aplicación del enfoque de derechos al cuidado para reconocer que toda persona tiene derecho a cuidar, a ser cuidado y a cuidar(se) o autocuidado ${ }^{(10)}$. La naturaleza innovadora de este reconocimiento es precisamente que, al ser un derecho universal, está desvinculado de la relación asalariada formal y las consiguientes medidas de conciliación trabajo-familia cuando existen -acotadas mayoritariamente a trabajadoras mujeres madres y muy pocas veces a los padres- o del estado de necesidad -por condición de vulnerabilidad socioeconómica, de género, étnica, etaria-, para situarse como un derecho humano individual, universal e inalienable de cada persona. Esta consagración tuvo su impulso gracias a la incorporación en las agendas de los mecanismos para el adelanto de la mujer, que en el marco de las Conferencias Regionales sobre la Mujer de América Latina y el Caribe, promovieron los consensos necesarios para aplicar el enfoque de derechos al cuidado y poder volcarlo, en algunos países, a la normativa nacional y los instrumentos internacionales. En este sentido, la División de Asuntos de Género de la Comisión Económica para América Latina y el Caribe (CEPAL) impulsó fuertemente tal reconocimiento, que fue adoptado en tres consensos regionales: el Consenso de Quito de 2007, el Consenso de Brasilia de 2010, y el Consenso de Santo Domingo de 2013, adoptados en las respectivas conferencias regionales sobre la mujer ${ }^{(11)}$. En octubre de 2016, en el marco de la XIII Conferencia Regional de la Mujer, se sumó un nuevo consenso denominado "Estrategia de Montevideo para la Implementación de la Agenda Regional de Género en el Marco del Desarrollo Sostenible hacia 2030", el cual fortalece los logros alcanzados y ratifica la importancia del cuidado como derecho.

Cabe señalar que el derecho a la alimentación adecuada no tracciona para establecer la necesaria vinculación con el cuidado. A su vez, los reclamos en torno a la problemática del cuidado, en toda su complejidad, no perciben la potencialidad que tienen ambos derechos -en tanto derechos humanos y no derechos para las mujeres a que cuiden o alimenten mejor a sus hijos e hijas o a sus 
adultos mayores- para operar como una herramienta potente para un cambio en las condiciones de su ejercicio, con un impacto considerable en las políticas públicas.

En este artículo, busco explorar el compromiso de pasar de la retórica de derechos a un accionar estatal que haga visible las tensiones actuales, de modo de superar posiciones refractarias al reconocimiento de la necesaria transversalidad de las políticas públicas basadas en derechos. Por un lado, analizo el alcance del derecho a la alimentación que tiene una traducción normativa amplia pero un escaso impacto a nivel de institucionalidad pública y, por el otro, el reconocimiento reciente del cuidado como derecho, especialmente a nivel del sistema interamericano de derechos humanos, y la invisibilización persistente en las políticas públicas. Claro está que la demandada transversalidad requiere también de la incorporación de un enfoque de género que haga visibles las asimetrías de poder entre ambos sexos y las consiguientes construcciones culturales que concentran la responsabilidad de la satisfacción del cuidado y de la alimentación en las mujeres. Las conclusiones dejarán abierta una agenda de investigación y acción que identifica tensiones y oportunidades para avanzar en el efectivo ejercicio de derechos humanos.

\section{DERECHOS $\mathfrak{i}$ (DES)ARTICULADOS?}

Ser titular del derecho humano a la alimentación incluye la posibilidad de que cada persona tenga acceso a una alimentación adecuada en cantidad y calidad suficientes, y que este acceso no se restrinja solamente a la ingesta de alimentos, sino que las políticas diseñadas para satisfacerlo deben hacerlo en toda su complejidad, destinando los recursos necesarios para poder ejercer este derecho a lo largo del ciclo de vida. Claro está que esta definición se orienta, principalmente, a garantizar la dinámica cotidiana en torno a la alimentación, que incluye la provisión de nutrientes "adecuados" y donde organismos especializados como la Organización de Naciones Unidas para la Agricultura y la Alimentación (FAO) o la Organización Panamericana de la Salud (OPS) aportan los estándares y niveles de calidad, cantidad, adecuabilidad y adaptabilidad de los alimentos, en sus distintos ciclos. Al respecto, la FAO adoptó 17 directrices voluntarias para apoyar los esfuerzos estatales para lograr "la realización progresiva del derecho a una alimentación adecuada en el contexto de la seguridad alimentaria nacional"(12,13).

Sin embargo, y a pesar de la relevancia de los debates en torno a la seguridad alimentaria y las propuestas de soberanía alimentaria, poco o nada se dice del debate "puertas adentro"(14). Esto es, cómo se ejerce cotidianamente un derecho "adecuado" en las condiciones "no adecuadas" en que se resuelve el cuidado al interior de los hogares en América Latina. En otros términos: todos y todas tenemos derecho a una alimentación adecuada; sin embargo, este reconocimiento a las posibilidades de proveer(nos) este alimento, en sus distintos momentos de transformación de la materia prima, no debe recaer unilateralmente en una mujer, quien termina siendo solo identificada como "la madre" y responsable de todos los problemas vinculados con una deficiente alimentación. Incluso, alcanza niveles cuasi punitivos en los casos de muchos de los programas de transferencias condicionadas de ingresos vigentes en la región: cuando las madres asisten con sus niños, niñas y adolescentes a los controles de salud son reprendidas y/o amenazadas por profesionales de la salud -desde pediatras, nutricionistas, hasta trabajadoras sociales- debido a que los déficit nutricionales de sus hijos e hijas serían responsabilidad de estas agobiadas madres "por no procurar una ingesta saludable". En ningún momento se asocia cuidado con trabajo, ni cuidado con alimentación, ni ambos con la injusta organización social del cuidado y del trabajo, sino que las exigencias son impuestas a las mujeres por "todo lo que no hicieron o hicieron mal" en el marco de una escasísima o mísera transferencia monetaria, la cual está condicionada a que cumplan con estos controles ${ }^{(15)}$. 
En rigor, si vinculamos la semántica de alimentación y cuidado, se implican mutuamente, cargando de connotaciones valorativas al cuidado que demanda alimentar(se), sin mirar la distribución de responsabilidades en torno a este. Y claro está que conceptos vinculados a la calidad o adecuabilidad son aplicados de manera asistencialista sobre los efectos que produce en la persona, totalmente desvinculados de los procesos de producción y distribución. Los aportes desde el enfoque de género y la economía del cuidado no solo no han ingresado en la corriente central del desarrollo sino que, por el contrario, la dinámica cada vez más asistencialista de muchas de las acciones y programas en el campo de las políticas sociales a nivel regional dan cuenta de ello.

De manera similar ocurre con el enfoque de derechos, que presenta altos niveles de invocación política y escasa traducción empírica. Tal como demanda esta metodología, existen numerosas interpretaciones del alcance del concepto alimentación adecuada. Así, el Comité del Pacto Internacional de Derechos Económicos, Sociales y Culturales (PIDESC), ha señalado que el derecho a la alimentación:

...se ejerce cuando todo hombre, mujer o niño, ya sea sólo o en común con otros, tiene acceso físico y económico, en todo momento, a la alimentación adecuada o a medios para obtenerla [...] El contenido básico del derecho a la alimentación comprende [...] la disponibilidad de alimentos en cantidad y calidad suficientes para satisfacer las necesidades alimentarias de los individuos, sin sustancias nocivas, y aceptables para una cultura determinada; la accesibilidad de esos alimentos en formas que sean sostenibles y que no dificulten el goce de otros derechos humanos... ${ }^{(16)}$

Asimismo, el órgano de monitoreo del Pacto de derechos sociales del sistema internacional de derechos humanos considera que este derecho tiene cuatro elementos específicos, el primero es la adecuabilidad, que implica no solo cantidad sino, principalmente, calidad, en cualquiera de las etapas de la vida en la que se encuentra la persona, además de la relevancia del respeto por los patrones culturales, sociales, étnicos de la persona. Los siguientes elementos refieren a la disponibilidad, a la accesibilidad (económica y física), con particular atención a las personas con mayor vulnerabilidad $y$, finalmente, la sostenibilidad, íntimamente vinculada al concepto de seguridad alimentaria. En la misma dirección, la FAO considera que se alcanza el carácter de alimentación adecuada cuando:

La ingesta de comida diaria posee todos los requerimientos nutricionales, tanto cuantitativos (contenido energético) como cualitativos (proteínas, vitaminas y contenido mineral). La comida es segura para ser consumida por seres humanos y no provoca enfermedades. La comida es culturalmente aceptable para aquellos que la consumen. [Traducción del original: Daily food intake meets all nutritional requirements, quantitatively (energy content) and qualitatively (protein, vitamins and minerals content). The food is safe for human beings to eat and does not cause any disease. The food is culturally acceptable by those who consume it. ${ }^{(17)}$

Los estándares interpretativos continúan y se articulan entre sí en relación con esfuerzos por precisar el alcance del derecho a la alimentación, pero comienzan a desarticularse en relación con el cuidado. Así, en materia de cuidado poco se habla sobre ¿qué es lo "adecuado"? ¿El tiempo, la infraestructura, el dinero, la interdependencia de actores, la calidad, la cantidad, el afecto? ¿Todos estos elementos conforman lo "adecuado" para el cuidado o se requieren más? ¿Cómo se mide la disponibilidad y sostenibilidad del cuidado?

A modo de ejercicio, si se analiza las responsabilidades de cuidado establecidas por la Convención sobre los Derechos del Niño, son los padres o adultos responsables del niño, niña y adolescente los garantes primordiales de la provisión de todo aquello relacionado con medios económicos y condiciones de 
vida necesarias, siempre "considerando sus posibilidades". Claramente la norma señala a ambos progenitores como responsables de todo lo vinculado con el cuidado; sin embargo, en la práctica, son principalmente las mujeres quienes se ocupan de los niños, niñas y adolescentes.

Otro ejemplo más contundente aún de reconocimiento explicito del derecho al cuidado se ha consagrado en la Convención Interamericana sobre la Protección de los Derechos Humanos de las Personas Mayores, aprobada por la Asamblea General de la Organización de los Estados Americanos en 2015 y que entró en vigor en diciembre de 2016, la cual reconoce en su Art. 12 que cada persona adulta mayor tiene:

...derecho a un sistema integral de cuidados que provea la protección y promoción de la salud, cobertura de servicios sociales, seguridad alimentaria y nutricional, agua, vestuario y vivienda; promoviendo que la persona mayor pueda decidir permanecer en su hogar y mantener su independencia y autonomía. ${ }^{(18)}$

Y para la provisión, reconoce de modo explícito que es el Estado quien tiene la obligación de:

...diseñar medidas de apoyo a las familias y cuidadores mediante la introducción de servicios para quienes realizan la actividad de cuidado de la persona mayor, teniendo en cuenta las necesidades de todas las familias y otras formas de cuidados, así como la plena participación de la persona mayor, respetándose su opinión. ${ }^{(18)}$

De este modo, la Convención recoge manifiestamente el enfoque de derechos, y enfatiza la adopción de respuestas integrales, señalando en el mismo artículo que:

Los Estados Parte deberán adoptar medidas tendientes a desarrollar un sistema integral de cuidados que tenga especialmente en cuenta la perspectiva de género y el respeto a la dignidad e integridad física y mental de la persona mayor. ${ }^{(18)}$

Es decir, a nivel regulatorio se constata un avance en torno a la interdependencia de derechos y responsabilidades. A su vez, el reconocimiento como receptores de cuidado y de autocuidado de las personas adultas implica un paso fundamental en el ejercicio de derechos ya que, a la fecha, la mayoría de los ordenamientos nacionales los incluían solo si habían tenido una incorporación formal a la seguridad social durante su vida activa, con prestaciones en salud y previsionales, pero si se habían desempeñado de manera informal o en el caso de las mujeres a cargo de tareas en el hogar, requerían de una determinación posterior asistencial del ámbito no contributivo o de la dependencia del esposo. De allí que, enmarcado en titulares de derechos a ejercer el cuidado, el espectro de obligaciones cambia notoriamente. Sin embargo, el interrogante que surge de cara a la futura entrada en vigor de la Convención es ¿cómo será recepcionado este mandato para los Estados? ¿La integralidad estará puesta en el diseño de sistemas de cuidado -similares a los establecidos en Uruguay o Costa Rica- o se seguirán reproduciendo lógicas estancas en la resolución del ciudado y, con ello, de la alimentación?

Lo expuesto hasta el momento remite nuevamente a la asimétrica e injusta organización social del cuidado, que estratifica la provisión de alimentos pero no solo referido a la accesibilidad sino a la calidad y adecuabilidad cultural de estos, a las posibilidades de acceder a la infraestructura necesaria para su provisión y otros elementos asociados, dejando a las mujeres $-y$ en algunos casos a las familias- ante la falta de oportunidades de acceder a las prestaciones de cuidado. Sin duda que esta situación va a tener impacto diferenciado: si se dispone de ingresos suficientes, se puede resolver de mejor manera; en cambio, cuando estos no existen, se acude a la oferta pública no estatal disponible: organizaciones de la sociedad civil, instituciones religiosas, voluntariado que se encarga del 
cuidado, programas asistenciales y familiares a cargo -abuelas y abuelos, tíos y tías solteros, hermanas mayores- dando cuenta de una enorme inestabilidad en las estrategias de cuidado ${ }^{(19,20)}$. Cabe señalar, por otra parte, que esta oferta también se resuelve con el trabajo de otras mujeres, y dada la interdependencia con otros derechos, de igual manera sucederá con la alimentación.

Sin embargo, no se debe caer en un simple reduccionismo de la problemática: si bien la disponibilidad de ingresos juega un papel central en la díada alimentación-cuidado, no necesariamente lo soluciona debido a la asimetría imperante en la sociedad. Gran parte de la fuerza explicativa $-y$ sin dudas reivindicativa- del enfoque de género es denunciar de qué manera el cuidado y la alimentación han sido asignados histórica y culturalmente a las mujeres, sin que los procesos de cambio ocurridos hayan alterado este patrón.

La evidencia empírica disponible en la región muestra que la autonomía y la igualdad están lejos de ser una realidad de las mujeres. Muy por el contrario, una mejoría relativa en algunas áreas de autonomía de las mujeres -por ejemplo la disponibilidad de ingresos como indicador de autonomía económica- convive con el aumento de responsabilidades de cuidado, y un número de hijos e hijas determinante y condicionante de la trayectoria laboral, situación que no afecta de igual manera a los varones.

En América Latina, los hogares pertenecientes a los primeros deciles de ingresos, los de menores recursos, tienen mayor número de miembros dependientes del cuidado (como niños, niñas y adolescentes, personas con discapacidad o enfermedades crónicas), por lo que mujeres de estos hogares tienen bajo su responsabilidad un número importante de personas dependientes y una demanda de tiempo de trabajo doméstico y de cuidados que limita la búsqueda de empleo y las posibilidades de inserción y permanencia en el mercado de trabajo. En el primer quintil de ingresos, un $42,3 \%$ de las mujeres mayores de 15 años no tienen ingresos propios; este porcentaje disminuye al $18,6 \%$ en el quinto quinti ${ }^{(21)}$.
El hecho de que una de cada tres mujeres de América Latina no posea ingresos propios $^{(21)}$ se posiciona como un dato ineludible a la hora de responsabilizar con más trabajo a las mujeres. A ello se adiciona que cuando las mujeres ingresan al mercado de trabajo lo hacen, mayoritariamente, en puestos de baja calificación y peor remunerados, al tiempo que persiste una brecha salarial que afecta a las mujeres. Por otra parte, las situaciones de violencia contra las mujeres vulneran el ejercicio de sus derechos y lejos está la región de haber avanzado de manera contundente en garantizar una vida libre de violencia para las mujeres y las niñas, tal como se obligaron 32 Estados de la región al ratificar la Convención para Prevenir, Sancionar y Erradicar la Violencia contra la Mujer "Convención de Belem do Pará".

El significativo paso que representó el reconocimiento del cuidado como derecho no se ha traducido aún con la fuerza necesaria para que trascienda esta estratificación y lleve a una problematización sobre las discriminaciones existentes. No se trata de recomendar un mayor esfuerzo de las madres sobre la comensabilidad o la nutrición deseable, sino precisamente cuestionar por qué se le transfiere toda la responsabilidad del ciclo de vida, dado que todas las personas demandan alimentos y cuidados a lo largo de toda su vida. Esto incluye acuerdos intergeneracionales, quedando de lado los intrageneracionales que se delegan solo a las mujeres, y de los cuales los varones quedan eximidos de tal responsabilidad. En los hechos, los varones proactivos en esta dirección pasan a ser los "buenos ejemplos" para mantener el statu quo del modelo patriarcal.

De cara a las evidencias disponibles, volvemos al inicio: ¿cómo se garantiza la interdependencia e indivisibilidad en el ejercicio de derechos? ¿Por qué la universalidad no se resuelve con un aumento de cobertura de las políticas sociales? ¿Es posible articular alimentación y cuidado desde la respuesta estatal? 


\section{Lejos de los enfoques: la traducción en} políticas públicas

Un hecho relevante que debe destacarse es que el enfoque de derechos ha posibilitado un consenso regional y el compromiso de impulsar políticas de Estado fundadas en el reconocimiento de cada persona como sujeto de derechos y no como depositaria de acciones estatales de carácter asistencial. En ese último caso, la práctica habitual -aún no desterrada- es que si las personas "califican" como carentes en algún ámbito de la vida, sea de ingresos, capacidades o habilidades, alimentos o vivienda, son "asistidas" ingresando en el heterogéneo mundo de la vulnerabilidad. Sin embargo, los países de la región poco han avanzado en garantizar un marco de políticas basadas en derechos, entre otras razones, porque se invisibilizan los postulados aquí desarrollados.

A modo de ejemplo, se ha determinado que la condición básica para alcanzar el derecho a la alimentación es la seguridad alimentaria y nutricional, la cual se "logra cuando todas las personas, en todo momento, disponen de alimentos adecuados, tienen acceso a ellos y los utilizan satisfactoriamente para llevar una vida sana"(22), al tiempo que se ha reafirmado como deber de los Estados, de instituciones privadas y de la sociedad civil en general, promover instrumentos y mecanismos para garantizar la alimentación adecuada de la población, en un compromiso de acción adoptado en el marco de la Cumbre Mundial sobre la Alimentación ${ }^{(22)}$ y renovado en posteriores consensos. Sin embargo, muy débil ha sido la vinculación entre el estándar de accesibilidad y disponibilidad de alimentos y quien(es) resuelven el cuidado. Mucho menos se menciona el derecho que le corresponde a toda persona a ser cuidada en condiciones adecuadas. En este sentido, Marco ${ }^{(23)}$ realiza una interesante aproximación a la vinculación entre calidad aplicada a la educación y cuidado de niños, niñas y adolescentes en América Latina. Por otra parte, Batthyány, Genta y Perrotta $^{(24)}$ analizan los "saberes expertos" en torno al cuidado infantil, especialmente de profesionales de la salud, identificando enfoques diferenciados respecto a los componentes del "buen cuidado" o las claves del cuidado de calidad.

En términos de ejecución de la política, la situación es aún más grave ya que, salvo excepciones, en general cada programa social actúa de manera autónoma con los otros, sin establecer interacciones para dar respuestas conjuntas a una misma problemática. Así, programas nutricionales tienen poca vinculación institucional con programas de salud o de empleo, como tampoco establecen áreas de trabajo común con el sector educación o infraestructura, y menos aún se visibiliza la necesidad de cuidado. Como ejemplo paradigmático, iniciativas de huertas comunitarias o microemprendimientos productivos en áreas rurales no contemplan políticas de cuidado para niños no escolarizados, con lo cual el proceso de inserción de la mujer en estas iniciativas se encuentra "atravesada" por la imposibilidad de disponer de estrategias de cuidado para sus hijos pequeños, lo cual condiciona sin duda su "rendimiento", sumado a una escasa disponibilidad en el uso del tiempo para este desempeño. Estos ejemplos son innumerables y no cabe el argumento de carácter de innovación, porque la región lleva aplicando este tipo de medidas desde hace más de 30 años, con los mismos resultados y cambios parciales en las estrategias.

Claro, es posible que se recurra al artilugio argumentativo de que el derecho al cuidado es de reciente reconocimiento, pero no se puede decir lo mismo del derecho a la alimentación adecuada -inscripto desde mediados del siglo pasado como compromiso en el campo de los derechos humanos- que impone a los Estados obligaciones positivas y negativas que deben satisfacer y que son de cumplimiento inmediato y es exigible a los Estados y que involucra a todos los poderes del Estado (Ejecutivo, Legislativo y Judicial). Ahora bien, en qué medida se ha actuado en consecuencia con la obligación de utilizar al máximo los recursos disponibles, tal como está establecido en los tratados de Derechos Humanos. Nuevamente como 
ejemplo, ¿cuál es el porcentaje del gasto Público social destinado a la alimentación adecuada? En los casos que se dispone de esta información, se consignan únicamente ciertos niveles de ejecución de programas alimentarios pero nunca en vinculación con la producción y distribución de alimentos, con la accesibilidad y, mucho menos, con el cálculo de los aportes económicos del cuidado no remunerado. $\mathrm{Al}$ respecto, se ha abierto un escenario importante a partir de la definición de indicadores de progreso para monitorear el cumplimiento del Protocolo de San Salvador, que es el Protocolo adicional a la Convención Americana en materia de Derechos Económicos, Sociales y Culturales. Este protocolo reconoce el derecho a la alimentación adecuada, y el mecanismo de monitoreo solicita a los Estados que informen el grado de cumplimiento a partir de indicadores de progreso ${ }^{(25)}$.

En caso de violaciones al ejercicio al derecho a la alimentación, la situación es más grave aún, dado que las personas deben disponer de recursos jurídicos y políticos necesarios para exigir el cese inmediato de la vulneración de este derecho -lo cual implica disponer de garantías de acceso a la justicia- y es posible solicitar la reparación de daños y que se dispongan las medidas necesarias para evitar su reiteración. El aspecto a considerar es cuántas de estas sentencias se fundamentan en un efectivo reconocimiento del derecho a la alimentación, y la casi nula vinculación con el cuidado y menos aún con una perspectiva de género. Mucho menos se menciona la sobrecarga de trabajo para las mujeres que demanda el cuidado -con alimentación incluida- en su vida cotidiana, por lo que regresamos al punto de inicio: la ausencia de la incorporación del impacto de la división sexual del trabajo al interior del hogar como determinante de las asimetrías en torno a las responsabilidades de cuidado afecta y vulnera derechos, no solo de las mujeres sino de los distintos sujetos demandantes de cuidado. En rigor, el ingreso del enfoque de género debe ser a partir de su rol transformador y no como una sumatoria de "adecuaciones" respetuosas del género ${ }^{(26)}$.
De conformidad con la Convención sobre la eliminación de todas las formas de discriminación a la mujer (CEDAW), el Estado debe abstenerse de cualquier medida discriminatoria contra las mujeres en relación con el derecho a la alimentación adecuada, sin importar su edad, condición económica, etnia, raza, y debe actuar de conformidad con los artículos 12.2, sobre nutrición de las mujeres lactantes y embarazadas, y el 14.2.g, sobre el acceso a los recursos productivos por parte de las mujeres que habitan en áreas rurales. El imperativo del principio de igualdad y no discriminación es de aplicación inmediata e ineludible por parte del Estado e implica no solo obligaciones negativas, sino positivas (en materia de prevención y erradicación de todo tipo de discriminación).

En términos de ejecución, la metodología implícita de los enfoques de derechos y de género es claramente la transversalidad: se debe "atravesar" la dinámica productora y ejecutora de políticas públicas en los tres poderes del Estado. El aspecto débil es que debe quedar en claro que constituye un deber estatal respaldar su accionar en derechos, por lo tanto, los gobiernos deben cumplir con obligaciones de hacer y de no hacer (obligaciones positivas y negativas) sino que, además, deben garantizar eventualmente vías para rendir cuentas por su incumplimiento o cumplimiento deficitario, al mismo tiempo que deben proveer mecanismos para dirimir conflictos, los que generalmente se realizan en el ámbito del Poder Judicial. Este último aspecto es el que tiene menor visibilidad desde las políticas sociales ya que, en general, los decisores públicos no suelen advertir la importancia de los sistemas y políticas de acceso a la justicia y las instancias de reclamos de derechos, como componentes esenciales para enriquecer la participación social, la transparencia, fiscalización y, en definitiva, la efectividad de las propias políticas.

La experiencia acumulada en estos últimos treinta años en la región, entre recuperación democrática, políticas de corte neoliberal, políticas divergentes con los regímenes de bienestar, desintegración y 
fragmentación de políticas sectoriales, focalización en desmedro de la universalidad, ausencia de consideración de la alimentación como expresión cultural de las sociedades, invisibilidad del cuidado en toda su expresión, falta de identificación de la injusta división sexual del trabajo como componente central de la desigualdad, deben despejar toda duda que el camino correcto es el de integrar a partir de los enfoques señalados. Y no solo es políticamente correcto, sino como fue desarrollado, es un mandato establecido en pactos y tratados internacionales de Derechos Humanos, refrendado a nivel constitucional y que ha sido ratificado por la mayoría de los Estados de América Latina.

También resulta indispensable que se internalice que la región -y cada habitanteno resisten más ensayos, sino que demandan de manera urgente políticas que garanticen la igualdad, la no discriminación, el ejercicio de derechos y el reconocimiento de que no se puede seguir sosteniendo estos arreglos desintegrados e inequitativos, en los que la injusta organización social del cuidado y de la alimentación persisten y reproducen prácticas que niegan el ejercicio de derechos humanos.

\section{Articulando derechos, garantizando igualdad y universalizando prestaciones}

En América Latina resulta urgente definir políticas de corte universal, integrales e interdependientes, en las que se abandone la retórica y se efectivicen diseños institucionales, que de manera transversal, garanticen el ejercicio de derechos civiles, políticos y económicos, sociales y culturales en el marco de la igualdad y no discriminación por razones de género, identidad y diversidad sexual, etnia, población indígena, campesina y afrodescendiente. Sin embargo, la definición en sí misma que no socave las bases de la injusta división sexual del trabajo y del cuidado y que no busque transformar el orden patriarcal, será otro ensayo más que no dará ningún fruto, y que se sumará a una larga lista de frustraciones, las que lejos están de ser inocuas dado que condenan generación tras generación a arreglos inequitativos y discriminatorios.

No se desconocen los esfuerzos que los Estados han realizado en el campo de las políticas sociales en lo que va de este milenio, que marcan una diferencia estructural en término de infraestructura, inversión en el gasto público, cobertura en educación, salud y previsión social, disminución de la pobreza y mortalidad infantil. Sin embargo, la oferta sistemática y sostenida de cuidado es bajísima en toda la región, a lo que debe sumarse el incremento de las tasas de embarazo adolescente. Los déficit alimenticios, la persistencia de la desnutrición, los fenómenos como la obesidad en la pobreza dan cuenta de que los Estados no han internalizado que se encuentran obligados a responder por la satisfacción de los derechos garantizados, y que para ello tienen que destinar hasta el máximo de los recursos que dispongan, entre otros estándares que deben adoptar (universalidad, igualdad y no discriminación, progresividad en las acciones y no regresividad en los derechos, acceso a la información y a la justicia).

Precisamente, el enfoque de derechos subraya que la obligación de respetar exige, fundamentalmente, la adopción de medidas legislativas y jurisdiccionales que la efectivicen y que se ejecuten en el campo de la política pública. Sirva como ejemplo sencillo, el bajo impulso que han tenido las medidas legislativas de ampliación de las licencias para varones para que ejerzan el cuidado, o la implementación de licencias parentales de mayor extensión, las que además no han sido demandadas activamente por los varones. Si no se implementan políticas explícitas que distribuyan equitativamente la responsabilidades de cuidado sobre los hijos e hijas, de los progenitores o personas con discapacidad, entre los cónyuges, se seguirá fortaleciendo el modelo societal de cuidado y alimentación a cargo de las mujeres, convirtiendo una supuesta responsabilidad en una obligación exclusiva y excluyente. Siguiendo con la articulación de derechos, si madres y padres están excluidos del sistema de 
protección social y del mercado de trabajo, y padecen de ciertas enfermedades crónicas, la alimentación del niño o de la niña se verá amenazada, como también la propia salud de los padres, con la diferencia de que se verá más afectada la mujer, ya que asume mayor cantidad de responsabilidades, en especial las de cuidado de forma casi exclusiva y estas responsabilidades no se distribuyen equitativamente.

Es decir, la afectación de derechos va concatenada, y va a perturbar todo el ciclo de vida hasta tanto no se adopten medidas que redistribuyan las obligaciones y responsabilidades. Retomando el concepto de "diamante de bienestar", en América Latina cada arista se encuentra sostenida por mujeres: en las familias, centralmente y exclusivamente; del mismo modo en los ámbitos comunitarios; $y$, en el caso del mercado, la oferta mercantil de cuidado se encuentra feminizada, siendo peor remunerada y con escasos niveles protectorios. Los sesgos de género regulatorios del Estado también trasladan todo el peso a las mujeres: las políticas de conciliación trabajo-familia las responsabilizan por el cuidado, restringiendo al mínimo las posibilidades de los varones y, en el caso de las medidas de corte asistencial, las principales destinatarias de los programas sociales son mujeres a quienes se les exige una serie de condicionalidades para recibir las prestaciones $\sin$ ofrecerles respuestas de cuidado. No solo que todo recae sobre sus espaldas, a lo que se suman además de los hijos e hijas, el marido, hermanos y progenitores, sino que además lo "debe hacer bien", ya que corren el riesgo de que les suspendan prestaciones si, por ejemplo, no cumplen las condicionalidades de manera "adecuada". Una de las estrategias prioritarias a adoptar es desarmar la confluencia asistencialista establecida en la que las mujeres son consideradas como las principales articuladoras de las políticas asistenciales -en su rol de receptoras de programas y cuidadoras- ante la ausencia de una institucionalidad en relación con el cuidado tanto de los niños, niñas y adolescentes como de los adultos mayores.

En paralelo, la ausencia de políticas laborales integrales, sin un fortalecimiento de políticas de cuidado para trabajadoras y trabajadores, con marcos regulatorios totalmente sesgados por género, ha redundado en malas condiciones de trabajo remunerado que afectan sobre todo a las mujeres -particularmente en términos de su autonomía y trayectoria- y condicionan el cuidado que realizan de sus familiares. Más alarmante resulta aún la falta de interés estratégico que demuestran los decisores públicos por establecer interdependencias entre distintas áreas de políticas. Por ejemplo, en el derecho a ejercer el cuidado en condiciones de calidad y en el derecho a ser cuidado se pueden identificar un conjunto de derechos específicos ya reconocidos y que integran esta noción, como el derecho a una alimentación adecuada, el derecho a la salud, a la educación, el derecho a la protección de la seguridad social, a la vivienda, entre otros, contemplados en los instrumentos internacionales analizados ${ }^{(26)}$.

A lo largo de este artículo enfaticé que las respuestas estatales no solo están lejos de integrar o articular el ejercicio de derechos sino que, por el contrario, la resolución del cuidado y de la seguridad alimentaria se sigue realizando a "costa" del incremento del trabajo de las mujeres, sin que se haya avanzado de manera sustantiva en implementar un enfoque de derechos humanos. Si no se revisa y denuncia enfáticamente la injusta división societal y sexual del trabajo y del cuidado, se continuará desviando el debate sobre la desigualdad y convalidando injusticias y discriminaciones. 


\section{REFERENCIAS BIBLIOGRÁFICAS}

1. Aguirre P. La complejidad del evento alimentario. En: Piaggio L, Solans A, (comp.). Enfoques socioculturales de la alimentación: Lecturas para el equipo de salud. Buenos Aires: Akadia; 2014. p 4-13.

2. Pautassi L, Zibecchi C, (coord). Las fronteras del cuidado: Agenda, derechos e infraestructura. Buenos Aires: ELA-Editorial Biblos; 2013.

3. Rodríguez Enríquez C. La cuestión del cuidado ¿el eslabón perdido del análisis económico? Revista CEPAL. 2012;(106):23-36.

4. Rodríguez Enriquez C, Pautassi L. La organización social del cuidado en niños y niñas: Elementos para la construcción de una agenda de cuidados en Argentina. Buenos Aires: ADCCIEPP-ELA; 2014.

5. Razavi S. The political and social economy of care in a development context: Conceptual issues, research questions and policy options [Internet]. Geneva: UNRISD; 2007 [citado 10 mar 2016]. Disponible en: http://tinyurl.com/mtchgse.

6. Esping Andersen G. Fundamentos sociales de las economías postindustriales, Barcelona: Ariel; 2000.

7. Evers A, Pilj M, Ungerson C. Payments for care: a comparative overview Aldershot: Avebury; 1994.

8. Naciones Unidas. Pacto Internacional de Derechos Económicos, Sociales y Culturales [Internet]. 1966 [citado 5 mar 2016]. Disponible en: http://tinyurl.com/ze3nf4e.

9. Naciones Unidas. Objetivos de Desarrollo Sostenible [Internet]. 2015 [citado 7 mar 2016]. Disponible en: http://tinyurl.com/gwktrzt.

10. Pautassi L. El cuidado como cuestión social: un aproximación desde el enfoque de derechos. (Serie Mujer y Desarrollo $N^{\circ}$ 87). Santiago de Chile: CEPAL; 2007

11. Comisión Económica para América Latina y el Caribe, División de Asuntos de Género. Acuerdos Regionales [Internet]. s/f [citado 6 mar 2016]. Disponible en: http://tinyurl.com/jplmxe2.

12. Organización de las Naciones Unidas para la Alimentación y la Agricultura. Directrices voluntarias en apoyo de la realización progresiva del derecho a una alimentación adecuada en el contexto de la seguridad alimentaria nacional. Roma: FAO; 2005.
13. Carrasco M, Pautassi L. Diez años del Plan Nacional de Seguridad Alimentaria en Argentina: Una aproximación desde el enfoque de derechos. De Prácticas y Discursos: Cuadernos de Estudios Sociales. 2015;4(5):1-22.

14. Aguirre P, Díaz Córdova D, Polisher G. Cocinar y comer en Argentina hoy. Buenos Aires: FUNDASAP, Fundación Essen; 2015.

15. Pautassi L, Arcidiácono P, Straschnoy M. Condicionando el cuidado: La Asignación Universal por Hijo para la protección social en Argentina. Revista Íconos. 2014;(50):61-75.

16. Naciones Unidas, Oficina del Alto Comisionado para los Derechos Humanos. Observación general $N^{\circ}$ 12: El derecho a una alimentación adecuada (artículo 11) [Internet]. 1999 [citado 5 mar 2016]. Disponible en: http://tinyurl.com/jk4tkdu

17. Food and Agriculture Organization of the United Nations. Methods to monitor the Human Right to adequate food. Rome: FAO; 2009.

18. Organización de los Estados Americanos. Convención Interamericana sobre la Protección de los Derechos Humanos de las Personas Mayores (A-70) [Internet]. 2015 [citado 5 mar 2016]. Disponible en: http://tinyurl.com/jdjtxsr.

19. Pautassi L, Zibecchi C. La provisión de cuidado y la superación de la pobreza infantil: Programas de transferencias condicionadas en Argentina y el papel de las organizaciones sociales y comunitarias (Serie Políticas Sociales 15). Santiago de Chile: CEPAL, División de Desarrollo Social; 2010

20. Rico MN. Crisis del cuidado y políticas públicas: el momento es ahora. En: Rico $M$, Maldonado Valera C. (edit). Las familias latinoamericanas interrogadas. Hacia la articulación del diagnóstico, la legislación y las políticas (Serie Seminarios y Conferencias No 61). Santiago de Chile: CEPAL; 2011.

21. Comisión Económica para América Latina y el Caribe. Informe regional sobre el examen y la evaluación de la Declaración y Plataforma de Acción de Beijing y el documento final del vigesimotercer período extraordinario de sesiones de la Asamblea General (2000) en los países de América Latina y el Caribe (LC/L.3951), Santiago de Chile: CEPAL; 2015.

22. Organización de las Naciones Unidas para la Alimentación y la Agricultura. Declaración de Roma sobre la Seguridad Alimentaria Mundial y Plan de Acción de la Cumbre Mundial sobre la Alimentación. Roma: FAO; 1996. 
23. Marco Navarro, F. Regulando la calidad construimos igualdad: Normas para el cuidado y la educación inicial en América Latina. En: Gonzálvez Torralbo H, (comp). Diversidad familiar, cuidados y migración: Nuevos enfoques y viejos dilemas. Santiago de Chile: Ediciones de la Universidad Alberto Hurtado; 2015. p. 119-150.

24. Batthyány K, Genta N, Perrotta V. La dimensión de género en el saber experto en cuidado infantil. Psicología, Conocimiento y Sociedad. 2014;4(1):33-58.
25. Organización de los Estados Americanos. Protocolo de San Salvador [Internet]. 1999 [citado 5 mar 2016]. Disponible en: http://tinyurl.com/ h5m2gmj.

26. Pautassi L. La alimentación desde un enfoque de derechos: Problemas persistentes, oportunidades emergentes. En: Pautassi L, Zibecchi C, (comp). Respuestas Estatales en torno a la alimentación y al cuidado [Internet]. Ciudad de Buenos Aires: Observatorio del Derecho a la alimentación en América Latina y el Caribe; 2012 [citado 10 mar 2016]. Disponible en: http://tinyurl.com/hbdzorp.

\section{FORMA DE CITAR}

Pautassi LC. La complejidad de articular derechos: alimentación y cuidado. Salud Colectiva. 2016;12(4):621-634. doi: 10.18294/sc.2016.941.

Recibido: 6 de abril de 2016 | Versión final: 5 de agosto de 2016 | Aprobado: 7 de octubre de 2016

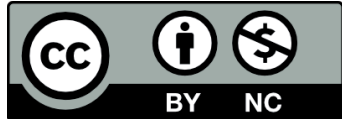

Este obra está bajo una licencia de Creative Commons Reconocimiento-NoComercial 4.0 Internacional. Reconocimiento - Permite copiar, distribuir y comunicar públicamente la obra. A cambio, se debe reconocer y citar al autor original. No Comercial - Esta obra no puede ser utilizada con finalidades comerciales, a menos que se obtenga el permiso. 